\title{
Using Mendelian inheritance errors as quality control criteria in whole genome sequencing data set
}

\author{
Valentina V Pilipenko ${ }^{1 *}$, Hua He ${ }^{1}$, Brad G Kurowski ${ }^{1,2}$, Eileen S Alexander ${ }^{3}$, Xue Zhang ${ }^{1}$, Lili Ding ${ }^{1,2}$, \\ Tesfaye B Mersha ${ }^{1,2}$, Leah Kottyan', David W Fardo ${ }^{4}$, Lisa J Martin ${ }^{1,2}$
}

From Genetic Analysis Workshop 18

Stevenson, WA, USA. 13-17 October 2012

\begin{abstract}
Although the technical and analytic complexity of whole genome sequencing is generally appreciated, best practices for data cleaning and quality control have not been defined. Family based data can be used to guide the standardization of specific quality control metrics in nonfamily based data. Given the low mutation rate, Mendelian inheritance errors are likely as a result of erroneous genotype calls. Thus, our goal was to identify the characteristics that determine Mendelian inheritance errors. To accomplish this, we used chromosome 3 whole genome sequencing family based data from the Genetic Analysis Workshop 18. Mendelian inheritance errors were provided as part of the GAW18 data set. Additionally, for binary variants we calculated Mendelian inheritance errors using PLINK. Based on our analysis, nonbinary single-nucleotide variants have an inherently high number of Mendelian inheritance errors. Furthermore, in binary variants, Mendelian inheritance errors are not randomly distributed. Indeed, we identified 3 Mendelian inheritance error peaks that were enriched with repetitive elements. However, these peaks can be lessened with the inclusion of a single filter from the sequencing file. In summary, we demonstrated that erroneous sequencing calls are nonrandomly distributed across the genome and quality control metrics can dramatically reduce the number of Mendelian inheritance errors. Appropriate quality control will allow optimal use of genetic data to realize the full potential of whole genome sequencing.
\end{abstract}

\section{Background}

Development of next-generation sequencing technologies has allowed for high-throughput genome sequencing. These advancements enable investigation of genetic association and linkage with high resolution; however, given the short read lengths in next-generation sequencing, error rates are much higher than traditional chip-based technologies [1]. Genotyping errors are a serious problem as an error rate as low as $1 \%$ to $2 \%$ can result in a false conclusion of linkage [2]. Several factors likely contribute to error rates, including sample preparation, sequencing platform variability, and sequence-specific characteristics. For example, certain areas of the genome are more likely

\footnotetext{
* Correspondence: valentina.pilipenko@cchmc.org

'Department of Pediatrics, Cincinnati Children's Hospital Medical Center,

Cincinnati, OH 45229, USA

Full list of author information is available at the end of the article
}

to be associated with errors caused by structural and functional complexity, such as repetitive sequences. In population-based samples, these errors are often detected when follow-up Sanger sequencing fails to validate calls. However, with family based data, Mendelian inheritance errors (MIEs) can help identify erroneous sequencing calls given that mutation occurs infrequently [3-5]. Although filters have been developed for whole genome sequencing (WGS) to identify regions of high complexity often associated with errors [1], there are no consensus guidelines for quality control procedures. Moreover, quantitative geneticists usually receive genotyping data in a very flexible and user-specified format called a VCF (variant call format) file. For instance, information related to quality controls (QCs) included in VCF files of the 1000 Genomes Project [6] differs from QC fields of VCF files used in the Genetic Analysis Workshop 18 
(GAW18) that were generated by Complete Genomics (Complete Genomics, Mountain View, CA). Thus, our goal was to identify the characteristics that determine MIEs and explore QC information provided by VCF files. To accomplish this, we used GAW18 data of chromosome 3 WGS family based data. We found that MIEs are associated with repetitive DNA sequences and that QC variable such as SVM (support vector machine) can reduce MIEs.

\section{Methods}

We analyzed sequence data of the human chromosome 3 obtained with DNA nanoarrays [7] generated by Complete Genomics. Variants listed in VCF file (VCFv4.0) were filtered to remove variants that didn't pass SVM (when SVM was less than zero; elements for SVM include allele balance, strand bias, fraction of bases with low quality, fraction of Mendelian errors) or INDEL5 filters, and those that had more than 1 alternative nucleotide. Passing status for each variant was provided in the PASS column of the VCF file. We extracted MIEs from the INFO column. The VCF file included a field for MIEs. MIEs were identified as part of the Complete Genomics workflow using SimWalk2 $[8,9]$. We also calculated MIE for binary variants using PLINK [10]. Although SimWalk2 utilizes all of the available family data and considers recombination and haplotypes to estimate MIEs, PLINK estimates MIEs using nuclear families in a single-locus manner. Given the MIE detection method in PLINK, it is expected that PLINK will identify fewer errors than SimWalk2. However, given its speed and ease of use, PLINK is often the preferred method for MIE estimation in a large data set. Because the number of alleles affects probability of MIE detections [11], a small fraction of nonbinary variants $(0.11 \%)$ was excluded from analysis to maintain homogeneous types of variants. The mean number of MIE per variant (MIE/ variant) was calculated by dividing the total number of MIEs by the total number of variants. We used a WaldWolfowitz runs test implemented in the $\mathrm{R}$ package "lawstat" [12] to assess if MIEs (sum of MIEs per 1000 variants) were randomly distributed. Sums of MIEs per 1000 variants were plotted against their genomic positions. From this plot we detected MIE peaks. Identified MIE peaks were assessed for complexity using the RepeatMasker [13] track of the UCSC Genome Table Browser [14]. We further reduced MIEs using an SVM filter, listed among $\mathrm{QC}$ variables in the VCF file.

\section{Results}

The uncleaned sequence data for the chromosome 3 is comprised of 1,757,461 variants. After removing variants that didn't pass SVM and INDEL5 filters, the number of variants were reduced by $8.5 \%$ to $1,607,227$. The second filtering procedure removed variants that had more than 1 alternative nucleotide resulting in reduction of variants by $0.1 \%$ to $1,605,431$. To examine the effect of secondstage filtering, we used the mean number of MIE per variant and number of variants with MIEs (Table 1). Importantly, the nonbinary variants (eg, variant with more than 1 alternative call) have a high rate of MIEs compared to biallelic variants. As such the remaining analyses include only binary variants.

For binary variants we also calculated MIEs using PLINK. The number of variants with MIEs calculated by PLINK was significantly lower than the number of variants with MIE provided by the VCF file $(14,886$ and 39,950 , respectively, $\mathrm{p}=2.20 \mathrm{E}-16$ ). Most of PLINK's MIEs were also flagged in the VCF file; only a small number of variants were identified by PLINK but not flagged in the VCF file (Figure 1).

MIEs were nonrandomly distributed regardless of the MIE detection method $\left(p\right.$ value $\left.<2.2 \times 10^{-16}\right)$. We identified 3 MIE peaks ( $\Sigma$ MIEs/1000 variants; Figure 2); Table 2 details the encoded transcripts located in these peaks. Overall, $49.26 \%$ of nucleotide bases in chromosome 3 comprise repetitive elements. Furthermore, MIE peaks were enriched for DNA repeats $(68.11 \%, 55.96 \%$, and $61.35 \%$ of repetitive sequence for peaks 1,2 , and 3 , respectively). To further explore the relationship between MIE and presence of repetitive sequences, we determined total number of MIEs variants located in repetitive regions compared to variants without MIEs (Table 3). Variants with MIEs were more often located in areas with repetitive sequences, regardless of MIE detection method ( $p$ value $<2.2 \times 10^{-16}$ ). Most repetitive elements were SINEs (short interspersed transposable elements, 31.5\%), LINEs (long interspersed transposable elements, $29.0 \%$ ), and LTRs (long terminal repeats, 13.8\%) (Figure 3).

To reduce the MIE rate, we explored a number of parameters provided in the INFO column and found that an SVM parameter threshold of $>3.5$ was most effective at reducing error (Table 4). Additional SVM filtering results of the runs test demonstrated that MIE distribution remained nonrandom regardless of MIE detection method $\left(p\right.$ value $\left.<3.6 \times 10^{-7}\right)$.

Table 1 MIE content in data sets where variants were based on secondary filtering criteria

\begin{tabular}{cccccc}
\hline Data sets & No. of variants & No. of MIE & MIE/variant & Range of MIE per variant & No. of variants with MIEs \\
\hline PASS & $1,607,227$ & 89,542 & 0.06 & $0-37$ & $41,489(2.6 \%)$ \\
Nonbinary & 1,796 & 23,995 & 13.36 & $0-37$ & $1,539(85.7 \%)$ \\
Binary & $1,605,431$ & 65,547 & 0.041 & $0-22$ & $39,950(2.5 \%)$ \\
\hline
\end{tabular}




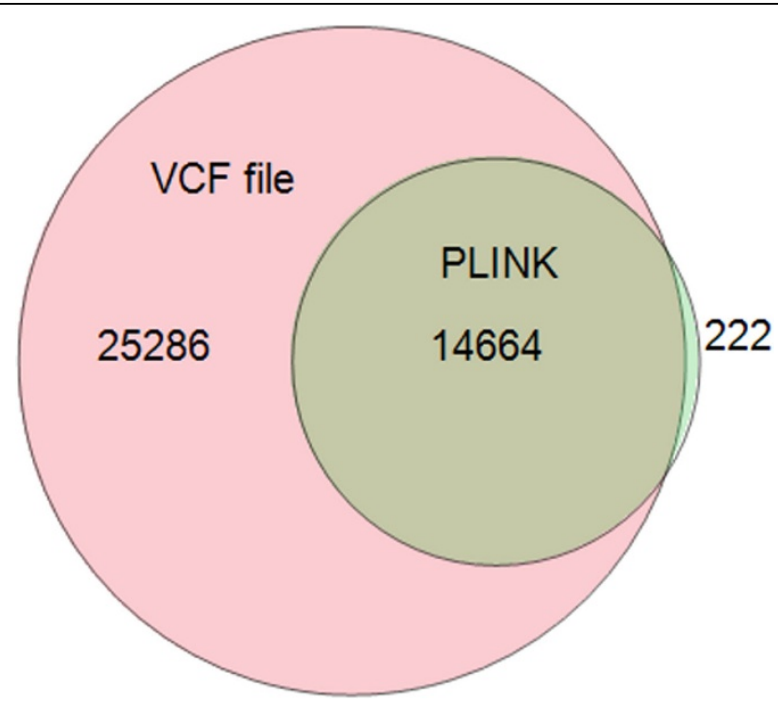

Figure 1 Venn diagram of MIEs extracted from the VCF file and detected by PLINK.

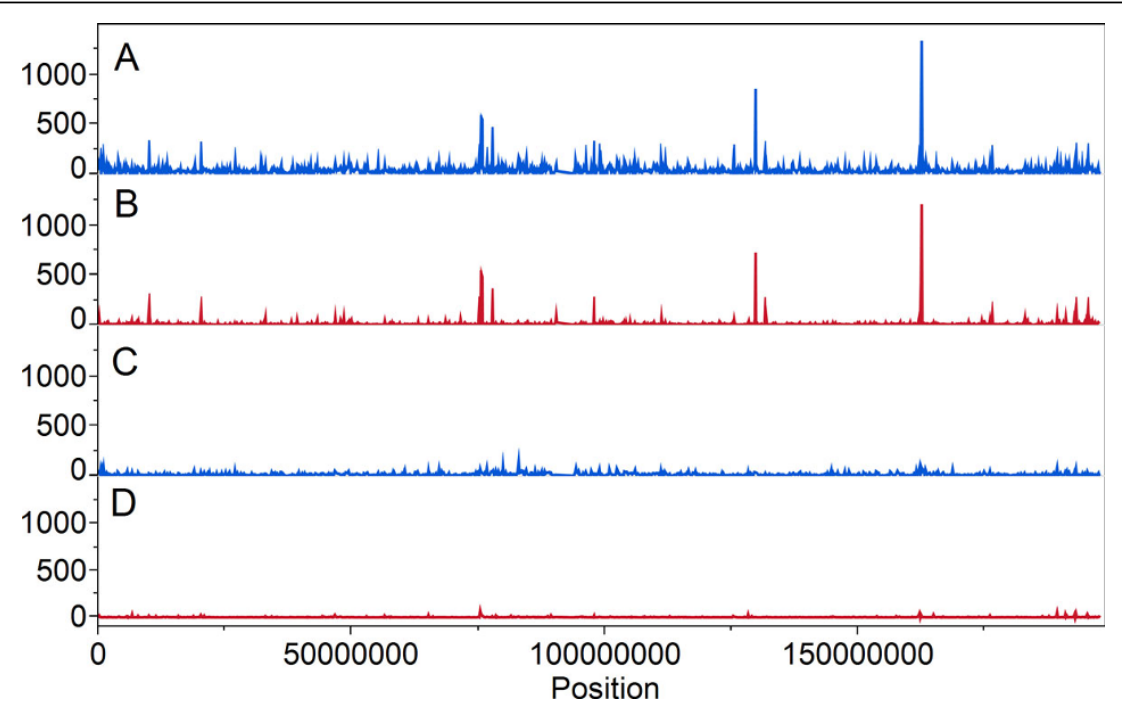

Figure 2 Distribution of MIEs over the chromosome $\mathbf{3}$ in $\mathbf{1 0 0 0}$ variant bins in the binary variants. A. Peaks of MIEs where variants passed SVM and INDEL5 filters. B. Peaks of MIEs calculated using PLINK. C. Subsequent filtering where variants passed additional SVM filter with threshold >3.5. D. Subsequent filtering where variants passed additional SVM filter with threshold $>3.5$ and MIE calculation using PLINK.

Table 2 Locations of the MIE peaks

\begin{tabular}{lll}
\hline Peak & Location, hg19 & \multicolumn{1}{c}{ Transcripts } \\
\hline 1 & $75,536,587-75,821,588$ & MIR1324, FRG2C, FLI20518, LOC401074, MIR4273, ZNF717 \\
2 & $129,767,883-129,837,072$ & ALG1L2, FAM86HP \\
3 & $162,441,039-162,675,707$ & Unknown \\
\hline
\end{tabular}

Table 3 Association of MIEs with areas in DNA sequences with repeats

\begin{tabular}{lccc}
\hline Way of MIEs detection & Variants & Located in repetitive areas & Located in area free from repeats \\
\hline From VCF file & with MIEs & 26745 & 13205 \\
& without MIEs & 836713 & 728768 \\
\hline PLINK & with MIEs & 10864 & 4022 \\
& without MIEs & 852594 & 737951 \\
\hline
\end{tabular}




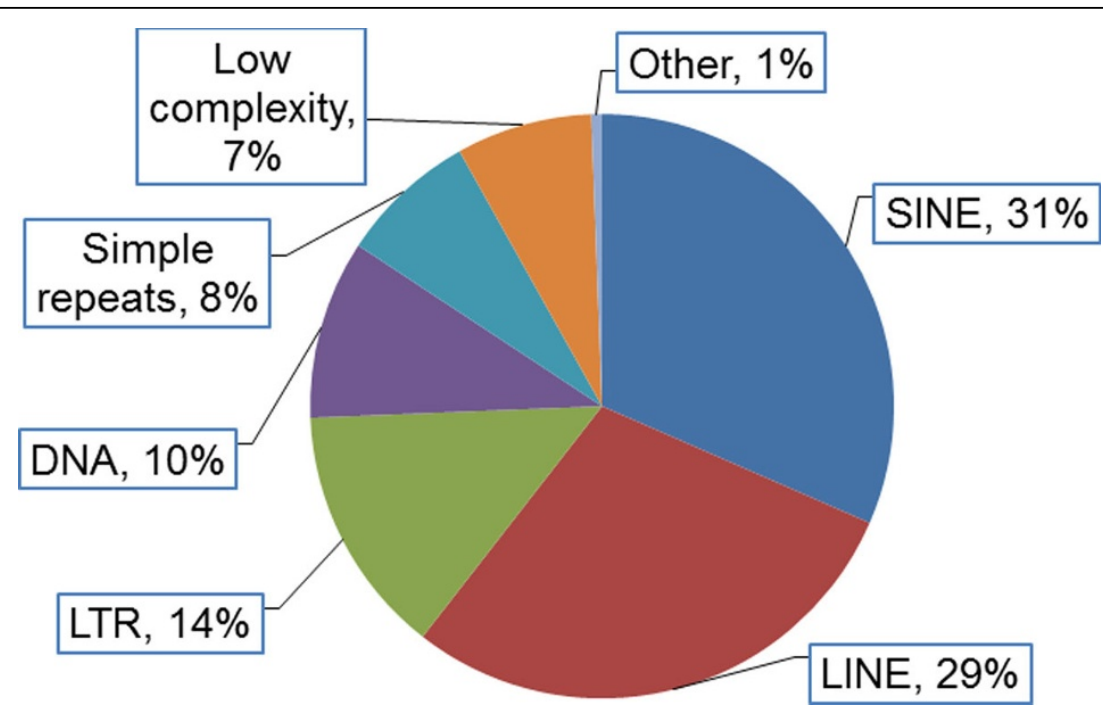

Figure 3 Types of chromosome 3 repetitive elements

Table 4 Reduction in MIE rate after employing SVM filter with different thresholds

\begin{tabular}{lccccc}
\hline Data sets & No. of variants & No. of MIEs & Mean no. of errors per variant & Range of MIEs per variant & No. of variants with MIEs \\
\hline SVM $>2$ & 1462201 & 40816 & 0.03 & $0-17$ & $25,706(1.76 \%)$ \\
SVM $>2.5$ & 1407230 & 33775 & 0.02 & $0-17$ & $22,094(1.57 \%)$ \\
SVM $>3$ & 1334454 & 26530 & 0.02 & $0-17$ & $18,324(1.37 \%)$ \\
SVM $>3.5$ & 1236087 & 19662 & 0.02 & $0-10$ & $14,511(1.17 \%)$ \\
SVM $>4$ & 1107983 & 13438 & 0.01 & $0-7$ & $10,629(0.96 \%)$ \\
\hline
\end{tabular}

\section{Discussion}

Through examination of MIEs, we demonstrated that MIEs were nonrandomly distributed over human chromosome 3, with several peaks enriched for errors. These peaks were localized in regions of repetitive sequence. Importantly, we found that using an SVM filter reduced MIEs.

The number of MIEs from PLINK was significantly lower than the number of MIEs flagged by Complete Genomics. These differences may be because PLINK calculates MIEs by dissecting a large pedigrees into nuclear families as compared to the MIEs from Complete Genomics which was based on extended families. However, distributions of MIEs exhibited a similar pattern between the two methods. Specifically, both methods identified 1 major and 2 moderate peaks occurring in a similar location. However, after SVM application of SimWalk2, there were more minor MIE peaks (see Figure 2C) than MIEs from PLINK (see Figure 2D), possibly as a result of the smaller number of variants with MIEs detected by PLINK.

Although Complete Genomics has a reported accuracy of greater than $99.999 \%$ [15], this accuracy is achieved after substantial data cleaning. Indeed, the uncleaned sequence data for chromosome 3 includes 1,607,227 variants passing SVM and INDEL5 filters. However, the cleaned data set contained 1,215,399 variants. The proprietary nature of the workflow doesn't describe filtering procedures used; removal of MIEs $(41,489)$ from the binary data set would not result in the actual reduction seen between the unclean and clean data set. Common parameters such as depth of coverage were not provided. Instead, we found that variants with more than 1 alternative nucleotide call have extremely high MIEs and should be considered suspect.

Using the binary set for analysis, we demonstrated that MIE peaks corresponded with regions of sequence complexity. Furthermore, we identified an SVM filter that significantly reduced MIEs. Although previous work on Complete Genomics data has suggested various filters to improve QC [16], an SVM filter was not applied. As an SVM work flow for genotype calling has been used for the 1000 Genomes Project [17] and the Exome Project [18], identifying SVM filters that improve data quality is important. The user-specific nature of VCF files highlights an important point that QC metrics may differ based on workflow. Thus, future studies will need to explore this important issue. 


\section{Conclusions}

In summary, examination of the areas with increased MIEs revealed that these areas were made up of repetitive sequence. Given that there is no consensus on filters to improve QC, identification of features associated with sequencing error will improve data quality and will allow optimal use of genetic data to realize the full potential of WGS studies.

\section{Competing interests}

The authors declare that they have no competing interests.

\section{Authors' contributions}

VP performed the statistical analysis and drafted the manuscript. LJM conceived the design of the statistical analysis. $\mathrm{HH}$ helped with statistical analysis. BGK, ESA, TMB, DWF, HH, XZ, LD, LK and LJM helped with the writing of the manuscript. All authors read and approved the final manuscript.

\section{Acknowledgements}

The Genetic Analysis Workshops are supported by NIH grant R01 GM031575 from the National Institute of General Medical Sciences. This work was supported in part by NIH grants 8P20GM103436-12 (DWF, KN), K25 AG043546 (DWF), NS36695 (LD, LJM), Al070235 (HH, LJM, TMB), Al066738 (LM), HL111459 (LJM, VP), T32-ES10957 (ESA), K12 HD001097-16 (BGK), K01HL103165 (TMB).

The GAW18 whole genome sequence data were provided by the T2DGENES Consortium, which is supported by NIH grants U01 DK085524, U01 DK085584, U01 DK085501, U01 DK085526, and U01 DK085545. The other genetic and phenotypic data for GAW18 were provided by the San Antonio Family Heart Study and San Antonio Family Diabetes/Gallbladder Study, which are supported by NIH grants P01 HL045222, R01 DK047482, and R01 DK053889. The Genetic Analysis Workshop is supported by NIH grant R01 GM031575.

This article has been published as part of BMC Proceedings Volume 8 Supplement 1, 2014: Genetic Analysis Workshop 18. The full contents of the supplement are available online at http://www.biomedcentral.com/bmcproc/ supplements/8/S1. Publication charges for this supplement were funded by the Texas Biomedical Research Institute.

\section{Authors' details}

'Department of Pediatrics, Cincinnati Children's Hospital Medical Center, Cincinnati, OH 45229, USA. ${ }^{2}$ Department of Pediatrics, University of Cincinnati College of Medicine, Cincinnati, OH 45229, USA. ${ }^{3}$ Department of Environmental Health, University of Cincinnati College of Medicine, Cincinnati OH 45229, USA. ${ }^{4}$ Department of Biostatistics, University of Kentucky, Lexington, KY 40356, USA.

Published: 17 June 2014

\section{References}

1. Reumers J, De Rijk P, Zhao H, Liekens A, Smeets D, Cleary J, Van Loo P, Van Den Bossche M, Catthoor K, Sabbe B, et al: Optimized filtering reduces the error rate in detecting genomic variants by short-read sequencing. Nat Biotechnol 2012, 30:61-68.

2. Abecasis GR, Cherny SS, Cardon LR: The impact of genotyping error on family-based analysis of quantitative traits. Eur J Hum Genet 2001, 9:130-134.

3. Saunders IW, Brohede J, Hannan GN: Estimating genotyping error rates from Mendelian errors in SNP array genotypes and their impact on inference. Genomics 2007, 90:291-296.

4. Kong A, Frigge ML, Masson G, Besenbacher S, Sulem P, Magnusson G, Gudjonsson SA, Sigurdsson A, Jonasdottir A, Wong WS, et al: Rate of de novo mutations and the importance of father's age to disease risk. Nature 2012, 488:471-475.

5. Veltman JA, Brunner HG: De novo mutations in human genetic disease. Nat Rev Genet 2012, 13:565-575.
6. Danecek P, Auton A, Abecasis G, Albers CA, Banks E, DePristo MA, Handsaker RE, Lunter G, Marth GT, Sherry ST, et al: The variant call format and VCFtools. Bioinformatics 2011, 27:2156-2158.

7. Drmanac R, Sparks AB, Callow MJ, Halpern AL, Burns NL, Kermani BG, Carnevali P, Nazarenko I, Nilsen GB, Yeung G, et al: Human genome sequencing using unchained base reads on self-assembling DNA nanoarrays. Science 2010, 327:78-81.

8. Sobel E, Lange K: Descent graphs in pedigree analysis:applications to haplotyping, location scores, and marker-sharing statistics. Am J Hum Genet 1996, 58:1323-1337.

9. Sobel E, Papp JC, Lange K: Detection and integration of genotyping errors in statistical genetics. Am J Hum Genet 2002, 70:496-508.

10. Purcell $S$, Neale B, Todd-Brown K, Thomas L, Ferreira MA, Bender D, Maller J, Sklar P, de Bakker PI, Daly MJ, et al: PLINK: a tool set for whole-genome association and population-based linkage analyses. Am J Hum Genet 2007, 81:559-575.

11. Douglas JA, Skol AD, Boehnke M: Probability of detection of genotyping errors and mutations as inheritance inconsistencies in nuclear-family data. Am J Hum Genet 2002, 70:487-495.

12. Hui W, Gel YR, Gastwirth JL: lawstat: An R Package for Law, Public Policy and Biostatistics. J Stat Softw 2008, 28:1-26.

13. Smit AFA, Hubley R, Green P: RepeatMasker Open-3.0 [http://www. repeatmasker.org].

14. Kent WJ, Sugnet CW, Furey TS, Roskin KM, Pringle TH, Zahler AM, Haussler D: The human genome browser at UCSC. Genome Res 2002, 12:996-1006.

15. Roach JC, Glusman G, Smit AF, Huff CD, Hubley R, Shannon PT, Rowen L, Pant KP, Goodman N, Bamshad M, et al: Analysis of genetic inheritance in a family quartet by whole-genome sequencing. Science 2010, 328:636-639.

16. Lam HY, Clark MJ, Chen R, Natsoulis G, O'Huallachain M, Dewey FE, Habegger L, Ashley EA, Gerstein MB, Butte AJ, et al: Performance comparison of whole-genome sequencing platforms. Nat Biotechnol 2012, 30:78-82.

17. Abecasis GR, Auton A, Brooks LD, DePristo MA, Durbin RM, Handsaker RE, Kang HM, Marth GT, McVean GA: An integrated map of genetic variation from 1,092 human genomes. Nature 2012, 491:56-65.

18. Exome Variant Server, NHLBI GO Exome Sequencing Project (ESP). Seattle, WA.

doi:10.1186/1753-6561-8-S1-S21

Cite this article as: Pilipenko et al:: Using Mendelian inheritance errors as quality control criteria in whole genome sequencing data set. BMC Proceedings 2014 8(Suppl 1):S21.

\section{Submit your next manuscript to BioMed Central and take full advantage of:}

- Convenient online submission

- Thorough peer review

- No space constraints or color figure charges

- Immediate publication on acceptance

- Inclusion in PubMed, CAS, Scopus and Google Scholar

- Research which is freely available for redistribution 